\title{
Dimensional crossover and band topology evolution in ultrathin semimetallic $\mathrm{NiTe}_{2}$ films
}

\author{
Joseph A. Hlevyack (iD ${ }^{1,2,10}$, Liang-Ying Feng ${ }^{3,10}$, Meng-Kai Lin ${ }^{1,2,10}$, Rovi Angelo B. Villaos ${ }^{3}$, Ro-Ya Liu ${ }^{1,2,4}$, Peng Chen ${ }^{5,6}$, Yao Li ${ }^{1,2}$,
} Sung-Kwan Mo $\mathbb{D}^{7}$, Feng-Chuan Chuang $\mathbb{B}^{3,8,9 凶}$ and T.-C. Chiang $\mathbb{D}^{1,2 凶}$

Nickel ditelluride $\left(\mathrm{NiTe}_{2}\right)$, a recently discovered Type-II Dirac semimetal with topological Dirac fermions near the Fermi energy, is expected to exhibit strong thickness-mediated electronic tunability and intrinsic two-gap superconductivity in the single-layer limit. Realizing such intriguing phenomena requires the fabrication of ultrathin $\mathrm{NiTe}_{2}$ films and an understanding of the underlying physics that is still under debate. By conducting experimental band mappings of ultrathin films prepared with molecular beam epitaxy, we reveal spectroscopic evidence for the dimensionality crossover of single-crystalline ultrathin $\mathrm{NiTe}_{2}$ films as a function of film thickness. As the film thickness increases from one to five layers, the gap in the conical topological surface states closes. Comparisons of experimental to first-principles results also highlight difficulties in fabricating atomically smooth single-layer NiTe 2 films. Our results not only provide further impetus for studying emergent phenomena in $\mathrm{NiTe}_{2}$ but also underscore the limitations of fabricating $\mathrm{NiTe}_{2}$ films for device applications.

npj 2D Materials and Applications (2021)5:40; https://doi.org/10.1038/s41699-021-00218-z

\section{INTRODUCTION}

Recent years have beheld the emergence of transition metal dichalcogenides (TMDCs) as a rich class of quasi-two-dimensional materials with electronic properties spanning from trivial semiconductors and semimetals to superconductors and topological phases $^{1-3}$. By virtue of the weak van der Waals bonding between layers, these materials can usually be isolated into single-layer phases $^{4-14}$ and can sometimes be perfect testing grounds for probing phenomena modified from or distinct from the bulk limit, including quantum spin Hall systems $s^{6,7}$, superconductivity ${ }^{8-10}$, charge density wave (CDW) phases ${ }^{11-13}$, and semiconductor-tosemimetal transitions ${ }^{14}$. Specific cases include a direct gap semiconductor phase in $\mathrm{MoS}_{2}$ and WS $\mathrm{W}_{2}$ in the single-layer limit ${ }^{4,5}$, $\mathrm{CDW}$ phases in single-layer $\mathrm{TiTe}_{2}$ and $\mathrm{VSe}_{2}$ distinct from bulk phenomena $^{12,13}$, and recently, a strong thickness-mediated Lifshitz transition from a semimetal to a semiconductor phase in ultrathin $\mathrm{PtTe}_{2}$ films ${ }^{14}$. The Ni-based TMDC NiTe $e_{2}$ is also ideal for exploring unusual phenomena in bulk and at reduced dimensions ${ }^{15-24}$. Though bulk $\mathrm{NiTe}_{2}$ is not superconducting, ab initio calculations have predicted an intrinsic two-gap superconducting state in single-layer $\mathrm{NiTe}_{2}$ with a transition temperature $T_{\mathrm{c}} \sim 5.7 \mathrm{~K}$ (ref. ${ }^{17}$ ). In addition, bilayer $\mathrm{NiTe}_{2}$ intercalated with an alkali metal may be a two-gap superconductor with a gap as high as $\sim 3.1 \mathrm{meV}$ and a critical temperature $T_{\mathrm{c}} \sim 11.3 \mathrm{~K}$ (ref. ${ }^{17}$ ). Moreover, recent work on $\mathrm{NiTe}_{2}$ under high pressure has shown the onset of superconductivity at small pressures and a second superconducting phase at even higher pressures with a transition temperature $T_{\mathrm{c}} \sim 7.5 \mathrm{~K}$ (ref. ${ }^{18}$ ), implying that $\mathrm{NiTe}_{2}$ is near the threshold of a superconducting instability. Ferromagnetic ordering at low temperatures with a Curie temperature $T_{\mathrm{F}} \sim 22 \mathrm{~K}$ has also been found in $\mathrm{NiTe}_{2}$ nanostructures ${ }^{20}$, but bulk $\mathrm{NiTe}_{2}$ itself is paramagnetic ${ }^{21}$.

Furthermore, $\mathrm{NiTe}_{2}$ is a candidate for the Type-II topological Dirac semimetal phase $\mathrm{e}^{21-24}$, in which low-energy excitations of emergent Dirac fermions generate a strongly tilted bulk Dirac cone that breaks Lorentz symmetry ${ }^{21-31}$. Such symmetry breaking should generate electronic and physical properties distinct from other topological semimetals, including an anisotropic magnetoresistance and chiral anomaly ${ }^{28-32}$ and unconventional superconductivity ${ }^{21,33}$. The topological nature of bulk $\mathrm{NiTe}_{2}$ single crystal has been addressed recently through both spin-resolved and conventional angle-resolved photoemission spectroscopy (ARPES), which revealed evidence for a topological Dirac cone with spin-polarized surface states at an energy $E \sim-1.4 \mathrm{eV}$ and a Type-II Dirac node near the Fermi level at $E=0 \mathrm{eV}$ (refs. ${ }^{23,24}$ ). The results contrast from the Pt- and Pd-based topological semimetals, which have Type-II Dirac cones located very far from the Fermi level at energies $E \sim-0.6 \mathrm{eV}$ to $-1.2 \mathrm{eV}$ (refs. ${ }^{25-29}$ ). Thus, in lieu of the Pt- and Pd-based systems, $\mathrm{NiTe}_{2}$ is likely an improved platform for exploiting topological Dirac fermions and other emergent phenomena for the development of spintronic or topological devices. However, to realize such physics, the thicknessdependent electronic band structure of ultrathin $\mathrm{NiTe}_{2}$ films needs to be fully addressed, a prerequisite for revealing the system's electronic tunability in the ultrathin-film limit and for further assessing the feasibility of fabricating atomically smooth $\mathrm{NiTe}_{2}$ films in a device setting.

Here, we present ARPES band mappings of ultrathin, semimetallic $\mathrm{NiTe}_{2}$ films of nominal thicknesses $1-5$ triatomic layers (TL) fabricated using molecular beam epitaxy (MBE) on bilayergraphene-terminated surfaces, which provide clear spectroscopic

\footnotetext{
'Department of Physics, University of Illinois at Urbana-Champaign, Urbana, IL, USA. ${ }^{2}$ Frederick Seitz Materials Research Laboratory, University of Illinois at Urbana-Champaign, Urbana, IL, USA. ${ }^{3}$ Department of Physics, National Sun Yat-sen University, Kaohsiung, Taiwan. ${ }^{4}$ National Synchrotron Radiation Research Center, Hsinchu, Taiwan. ${ }^{5}$ Shanghai Center for Complex Physics, School of Physics and Astronomy, Shanghai Jiao Tong University, Shanghai, China. ${ }^{6}$ Key Laboratory of Artificial Structures and Quantum Control (Ministry of Education), Shenyang National Laboratory for Materials Science, School of Physics and Astronomy, Shanghai Jiao Tong University, Shanghai, China. ${ }^{7}$ Advanced Light Source, Lawrence Berkeley National Laboratory, Berkeley, CA, USA. ${ }^{8}$ Department of Physics, National Tsing Hua University, Hsinchu, Taiwan. ${ }^{9}$ Physics Division, National Center for Theoretical Sciences, Hsinchu, Taiwan. ${ }^{10}$ These authors contributed equally: Joseph A. Hlevyack, Liang-Ying Feng, Meng-Kai Lin. ${ }^{凶}$ email: fchuang@mail.nsysu.edu.tw ; tcchiang@illinois.edu
} 
evidence of strong thickness-mediated electronic tunability of $\mathrm{NiTe}_{2}$ films. Specifically, our detailed band maps for increasing film thickness show the closing of the hybridization gap in the topological surface Dirac cone already at 5 TL in thickness and simultaneously, the development of linear-like bands near the Fermi level attributable to the lower branches of the Type-II Dirac cone in the bulk limit ${ }^{23,24}$, both indicative of the dimensionality crossover from the ultrathin-film to bulk-like regimes. Our results also suggest that $\mathrm{NiTe}_{2}$ is a robust semimetal even in the singlelayer limit, contrasting from the semiconducting behavior in $1 \mathrm{TL}$ $\mathrm{PtTe}_{2}$ (refs. ${ }^{14,34}$ ). Furthermore, first-principles calculations of $\mathrm{NiTe}_{2}$ based on density functional theory (DFT) are compared with the measured ARPES spectra to confirm the fabrication of singlecrystalline $\mathrm{NiTe}_{2}$ films in only the $1 T$ phase and thus to dismiss the question of another stable phase, such as the pyrite lattice ${ }^{35-37}$, appearing in our results. However, we discover that atomically smooth $\mathrm{NiTe}_{2}$ films grown with MBE on bilayer graphene are seemingly unstable in the ultrathin-film regime, raising potential serious questions about the feasibility of integrating smooth one $\mathrm{TL} \mathrm{NiTe}_{2}$ films in a device and highlighting possible difficulties in observing emergent phenomena in 1 and 2 TL systems.

\section{RESULTS AND DISCUSSION}

\section{Growth and characterizations of $1+2 \mathrm{TL}$ and $5 \mathrm{TL} 1 \mathrm{~T} \mathrm{NiTe}$}

$\mathrm{NiTe}_{2}$ has been suggested to exist in multiple lattice structures, including the $1 T$ and pyrite lattices (refs. ${ }^{15-24,35-37}$ ) (see Fig. 1a and Supplementary Fig. 1). We confirm that $\mathrm{NiTe}_{2}$ can adopt in theory four distinct phases: $1 \mathrm{~T}, 2 \mathrm{H}$, pyrite, and marcasite, whose lattice structures and corresponding bulk Brillouin zones all appear in Supplementary Fig. 1. Table 1 summarizes each of their normalized energy per unit cell, which reveals that the $1 T$ phase has the lowest energy, followed by the marcasite and pyrite lattices, and is thus the most energetically stable phase. Figure $1 \mathrm{a}$ presents the atomic lattice structure for $1 \mathrm{TL}$ of $1 \mathrm{~T} \mathrm{NiTe}{ }_{2}$, which contains three triangular atomic layers of Te and Ni stacked in the order Te-Ni-Te. The bulk lattice for $1 \mathrm{~T} \mathrm{NiTe}_{2}$ is assembled by vertically stacking many of these TLs directly on one another, each separated by a lattice constant $c=5.26 \AA$, with no other rotational and translational offsets, and van der Waals bonded with neighboring layers. The corresponding (001)-projected surface Brillouin zone is displayed in Fig. 1b. In this work, ultrathin $1 T$ $\mathrm{NiTe}_{2}$ films with thicknesses $N=1-5 \mathrm{TL}$ are grown on bilayergraphene-terminated $6 \mathrm{H}$-SiC $(0001)$ surfaces (BLG-SiC). Representative in situ reflection high-energy electron diffraction (RHEED) patterns for a BLG-SiC surface and a 5 TL film are shown in the left and right panels of Fig. 1c, respectively; the sharp diffraction streaks for the $5 \mathrm{TL}$ film are evidence of a highly well-ordered surface.

An overview ARPES spectra and a zoomed-in view of the same band map for a $1+2 \mathrm{TL} 1 \mathrm{~T} \mathrm{NiTe}_{2}$ film taken along the $\overline{\Gamma \mathrm{M}}$ direction, where " $1+2 \mathrm{TL}$ " means a film with a mixture of 1 and 2 TL ARPES signal, are displayed in the left and right panels of Fig. $1 \mathrm{~d}$, respectively. The corresponding calculated DFT band structure, consisting of the calculation for a 1 TL film (red curves) superimposed with that of a 2 TL film (blue curves), appears in Fig. 1e. Similar ARPES and theoretical results for a $5 \mathrm{TL}$ film, which we find are in the bulk-like regime, are summarized in Fig. $1 \mathrm{f}$ and $\mathrm{g}$, respectively. Evidently, the electronic band structure in Fig. 1d consists of a mixture of 1 and 2 TL signal, though the film thickness based upon the amount of deposition should be $1 \mathrm{TL}$. Incidentally, reduction of the calibrated film thickness from $1 \mathrm{TL}$ to $0.5 \mathrm{TL}$ in thickness, the former being the result shown in Fig. 1d, yields similar ARPES band maps as in Fig. 1d with faint bands arising from a 2 TL film thickness. For example, from the red theoretical curves in Fig. 1e, the band structure of $1 \mathrm{TL} 1 \mathrm{~T} \mathrm{NiTe}$ should be a relatively simple, robust semimetal with only three bands crossing the $\bar{\Gamma}$ point in the energy range $E=0$ to $-2 \mathrm{eV}$ and a negative gap of $\sim-0.50 \mathrm{eV}$ (see, for instance, Supplementary Fig. 4), quite unlike its Pt-based counterpart that is semiconducting at this thickness ${ }^{14,34}$. However, in the ARPES results in Fig. 1d, an additional weak band crosses at the $\bar{\Gamma}$ point at $E \sim-0.5 \mathrm{eV}$ (indicated by a red arrow in Fig. 1d), which can be ascribed to that of a 2 TL film thickness (compare to the blue curves in Fig. 1e). Furthermore, as the bands disperse along the $\overline{\Gamma M}$ direction away from the $\bar{\Gamma}$ point, additional bands not belonging to the $1 \mathrm{TL}$ system can be duly assigned to those of a $2 \mathrm{TL}$ film. The situation for the $5 \mathrm{TL}$ sample is a bit different from the $1+2 \mathrm{TL}$ system (Fig. 1f, g). Generally speaking, the first-principles calculation result in Fig. $1 \mathrm{~g}$ is more representative of the 5 TL ARPES map in Fig. $1 \mathrm{f}$ with the effects of any layer mixing less obvious at this thickness and is thus in accord with our expected thickness calibration. In other words, our results suggest that it is difficult to prepare a uniform $1 \mathrm{~T} \mathrm{NiTe}_{2}$ thin film on BLG-SiC, implying enhanced interlayer coupling relative to the Pt-based topological semimetals ${ }^{14}$. Since we observe $1+2 \mathrm{TL}$ and $5 \mathrm{TL}$ band structures in our ARPES results (see Fig. 1d, f), though we expect $1 \mathrm{TL}$ and $5 \mathrm{TL}$ signals, respectively, based on our thickness calibration, the surface energy of the BLG-SiC substrate, while conducive for fabricating other single-layer TMDC films ${ }^{6,7,10-14}$, may be too low for forming a uniform $1 \mathrm{TL} 1 \mathrm{~T} \mathrm{NiTe}$ film. Rather, the next TL starts forming over the first TL well before the BLG-SiC surface is fully covered. Clean layer-resolved evolution of the band structure using ARPES at film thicknesses such as 1 and 2 TL may thus be potentially problematic, which can complicate observation of emergent phenomena, such as two-gap superconductivity, in this thickness regime and possibly integration of $1 T \mathrm{NiTe}_{2}$ in a device setting.

\section{Gap opening in topological states at reduced thickness}

Despite these difficulties in realizing pure $1 \mathrm{TL}$ and $2 \mathrm{TL}$ systems, the results highlight a clear departure in the electronic band structures between films grown at low coverages and those in the bulk-like limit, which arises from quantum confinement along the surface normal. In particular, the band dispersions of the conical topological surface states (TSS) (refs. ${ }^{23,24}$ ), labeled as TSS in Fig. $1 \mathrm{~d}, \mathrm{f}$, are modified as the thickness is reduced from $5 \mathrm{TL}$ to $1+2$ TL. Figure $2 a$ displays the ARPES spectra taken along $\overline{\Gamma M}$ (left panel) and the same map zoomed-in around the TSS (right panel) for a $1+2 \mathrm{TL} 1 \mathrm{~T} \mathrm{NiTe}$, film; the corresponding second-derivative maps are displayed in Fig. 2b, whereas similar ARPES and secondderivative spectra for the $5 \mathrm{TL}$ film appear in Fig. $2 \mathrm{c}$ and $\mathrm{d}$, respectively. These results are very clear: At $1+2 \mathrm{TL}$ in thickness, a hybridization gap of $E_{\mathrm{g}} \sim 0.1 \mathrm{eV}$ opens in the conical TSS (right panels of Fig. 2a, b), typical of ultrathin topological films driven by quantum-mechanical coupling between the film's two boundaries $^{38,39}$, but for a 5 TL film, the gap in these surface states is not present within the experimental energy resolution (right panels of Fig. 2c, d). Furthermore, in the ARPES spectra for the 5 TL sample, linear bands that seemingly connect at or slightly above the Fermi level (identified by the label DB in the right panel of Fig. 1f) are present near the zone center, which in the bulk limit, are attributable to the lower branches of a bulk Type-II Dirac cone ${ }^{23,24}$, but for the $1+2 \mathrm{TL}$ film, these bands are entirely absent. The ARPES map for the 5 TL sample is also much more complicated than the $1+2$ TL film at the zone center, with a dense set of upside-down $U$-shaped bands appearing near the $\bar{\Gamma}$ point in the energy range $E=-0.25$ to $-1 \mathrm{eV}$ (Fig. $1 \mathrm{f}, \mathrm{g}$ ); these bands are mostly lacking in the $1+2$ TL case (Fig. 1d, e). Generally, the results for the $5 \mathrm{TL}$ sample are consistent with those previously reported for bulk $1 T \mathrm{NiTe}_{2}$ single crystal with a Type-II Dirac node near the Fermi energy ${ }^{23,24}$, though the film has not reached the bulk limit as evidenced by the discretization of the bulk occupied states into quantum well bands in the ARPES and theoretical results. 
$1 T \mathrm{NiTe}_{2}$ atomic structure

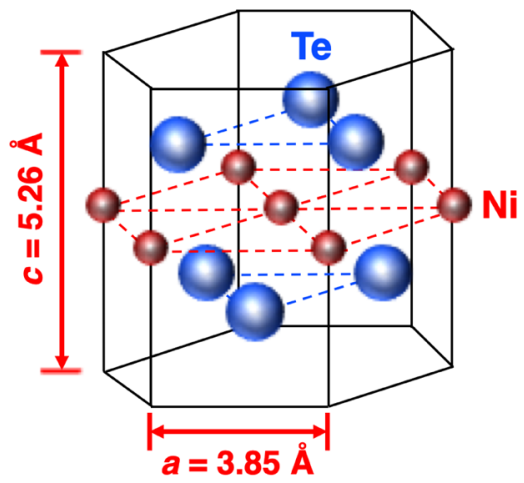

b

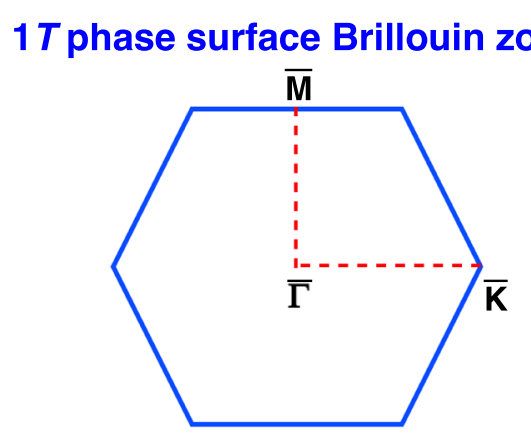

C

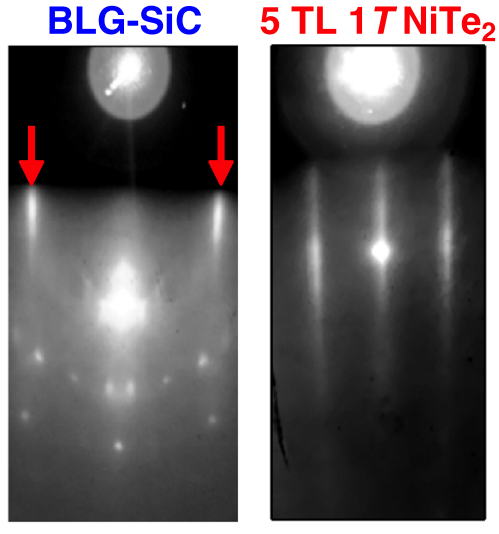

d
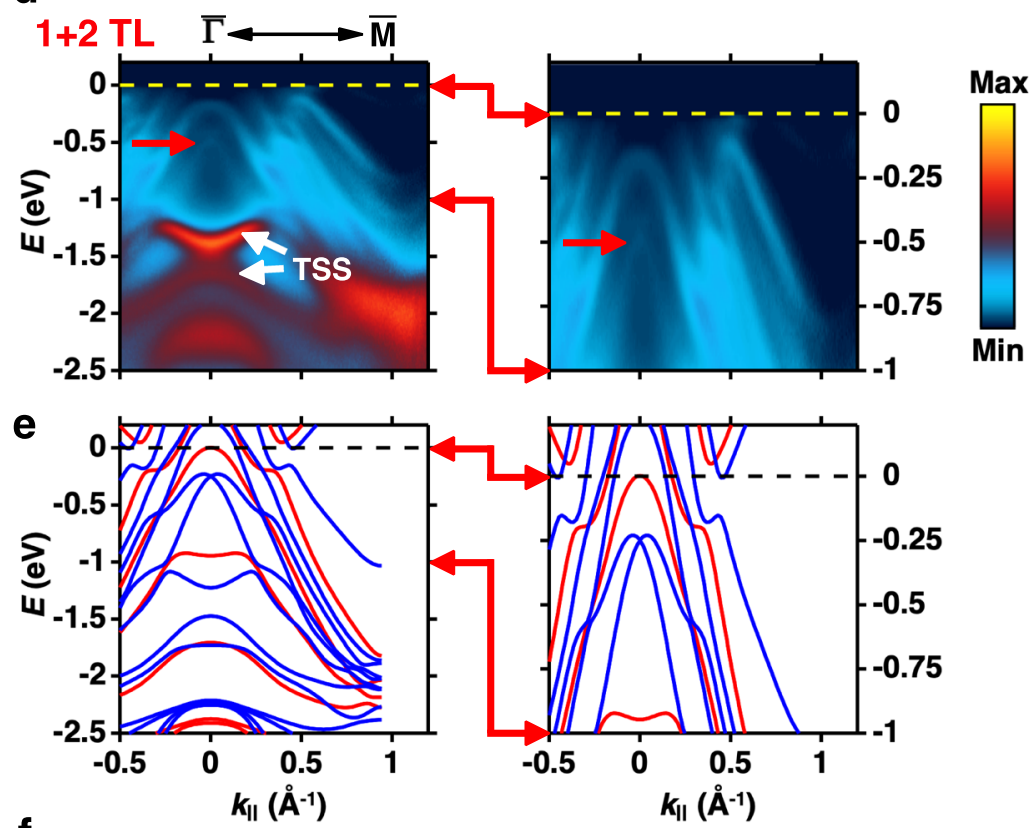

$\mathbf{f}$
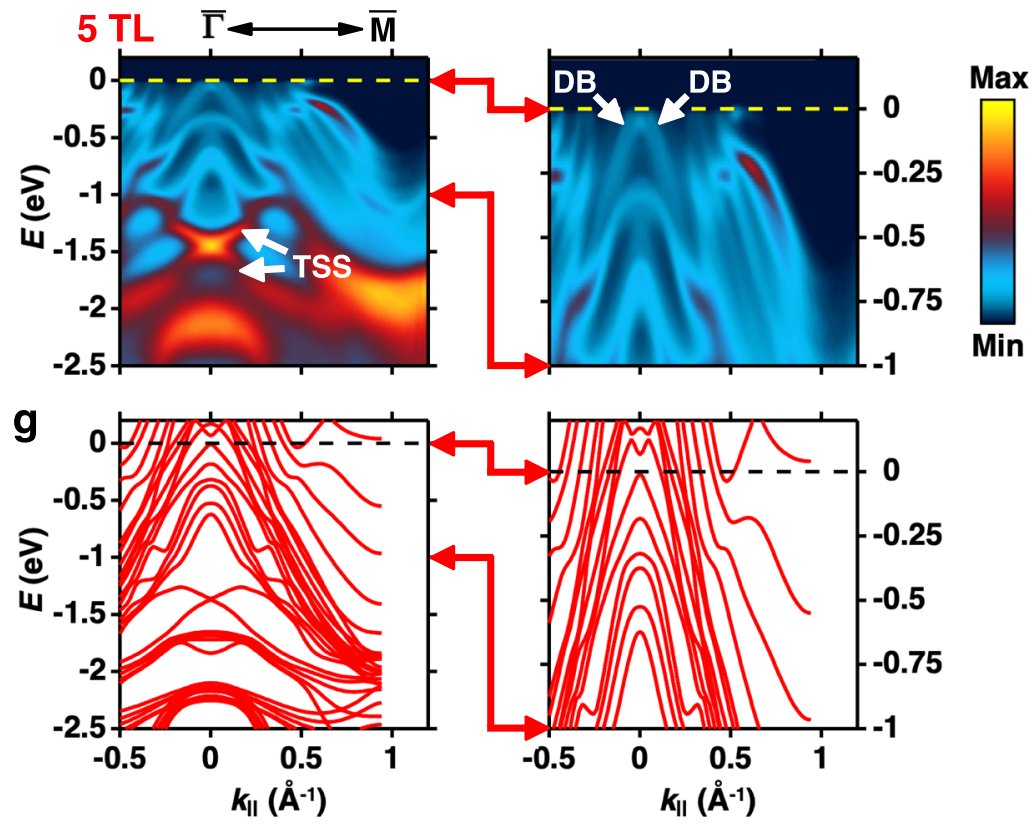

Fig. 1 Preparing and characterizing $1+2$ TL and 5 TL $1 T$ NiTe films. a Schematic diagram for the atomic lattice structure of $1 T$ NiTe $;$ the lattice constants are indicated. b Associated (001)-projected surface Brillouin zone for the $1 T$ phase. c Characteristic RHEED patterns of a BLG$\mathrm{SiC}$ sample (left panel) and a $5 \mathrm{TL} 1 \mathrm{~T} \mathrm{NiTe}$ sample (right panel); the red arrows in the RHEED pattern for BLG-SiC indicate the diffraction streaks due to bilayer graphene. d Overview ARPES spectra of $1+2 \mathrm{TL} 1 T \mathrm{NiTe}_{2}$ thin film (left panel) and the same band map zoomed-in near the Fermi level (right panel) taken with 21.218-eV photons at $30 \mathrm{~K}$; the red arrow identifies a band due to a $2 \mathrm{TL}$ film, whereas bands giving rise to the topological surface states in the bulk limit are labeled by TSS. e Corresponding calculated DFT band structure results for a 1 TL film (red curves) superimposed with those of a 2 TL film (blue curves). f ARPES band map of 5 TL $1 T \mathrm{NiTe}_{2}$ (left panel) and the same spectra zoomed-in near the Fermi level (right panel) measured with 21.218-eV photons at $30 \mathrm{~K}$; the labels TSS and DB identify the topological surface states and those bands belonging to the lower branches of the Type-II Dirac cone in the bulk limit, respectively. $\mathbf{g}$ Corresponding DFT calculations for a 5 TL film.

\section{Dimensionality crossover in the band maps of $1 \mathrm{~T} \mathrm{NiTe}_{2}$ films}

The thickness dependence of $1 T \mathrm{NiTe}_{2}$ films from the ultrathinfilm to bulk-like limit is of fundamental interest to reveal how the band structure of $1 \mathrm{~T} \mathrm{NiTe}_{2}$ evolves into a bulk Type-II Dirac semimetal system with topologically protected nontrivial states. ARPES band maps and their second-derivative spectra for ultrathin $1 T \mathrm{NiTe}_{2}$ films of nominal thicknesses $N=1-5 \mathrm{TL}$ taken along the
$\overline{\Gamma M}$ direction are summarized in Fig. $3 a$ and b, respectively. Figure $3 c$ displays the corresponding DFT calculations, whereas ARPES maps in Fig. $3 \mathrm{~d}$ are overlaid with the same theoretical results to foster further comparisons. A similar data set for the thicknessdependent band structure along the $\overline{\Gamma K}$ direction appears in Fig. 4. Some general comments about the bulk-like and ultrathin-film regimes are warranted for these results. None of these band maps 
show any evidence of other phases aside from $1 T \mathrm{NiTe}_{2}$ (see Supplementary Figs. 3 and 4), and all of these thin-film samples are semimetallic. For all thicknesses $<5 \mathrm{TL}$, the conical TSS at $E \sim$ $-1.45 \mathrm{eV}$ in the $5 \mathrm{TL}$ system are gapped. As the thickness increases from 1 to $5 \mathrm{TL}$, this hybridization gap becomes smaller and first closes at $5 \mathrm{TL}$ in thickness, signaling the onset of the bulk-like limit. A $5 \mathrm{TL}$ film is thus the critical thickness for realizing such a topologically protected surface Dirac cone with spin-polarized surface states ${ }^{23,24}$. As discussed previously, the $5 \mathrm{TL}$ film also shows incipient hints of the lower branches of the Type-II Dirac cone (Figs. 1f, 3, and 4). When the film thickness is reduced from $5 \mathrm{TL}$ to $4 \mathrm{TL}$, this structure mostly disappears, with fewer bands crossing the Fermi level near the zone center (compare Figs. 1f, 3, and 4); no bands cross the $\bar{\Gamma}$ point very close to the Fermi level for the $1+2$ TL and 3 TL samples, and the structure for the Type-II Dirac cone is entirely absent. Such observations alone are indicative of the strong thickness-mediated tunability of the band structure for $1 T \mathrm{NiTe}_{2}$.

The discussion can be generalized even further for other occupied bands in this system. Per the results in Figs. 3 and 4, an increase in the $1 T \mathrm{NiTe}_{2}$ film thickness from one thickness to the next necessarily results in the multiplication of quantum well bands, which is well-known in noble metallic films ${ }^{40}$. For example,

Table 1. Energy minimization DFT results for the stable lattice structures for $\mathrm{NiTe}_{2}$.

\begin{tabular}{llr}
\hline Lattice structure & \multicolumn{2}{c}{ Energy $(\mathrm{eV})$} \\
\cline { 2 - 3 } & Without SOC & With SOC \\
\hline $1 T$ & -49.6165 & -50.5413 \\
$2 H$ & -48.2776 & -49.1875 \\
Pyrite & -49.1255 & -50.0069 \\
Marcasite & -49.1325 & -50.0291 \\
\hline Here, the DFT calculations are presented with and without spin-orbit \\
coupling (SOC). The energy is normalized as 12 atoms (4 Ni + 8 Te) per \\
unit cell.
\end{tabular}

a

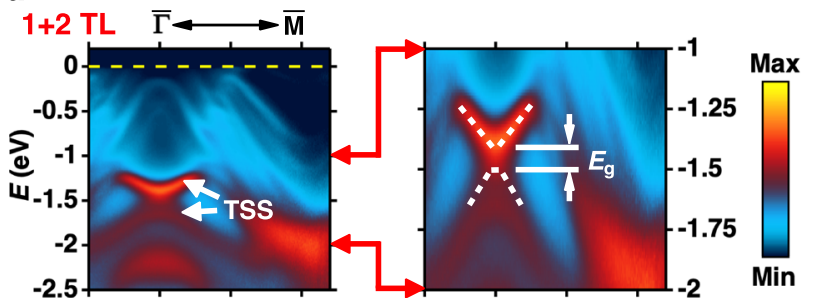

b

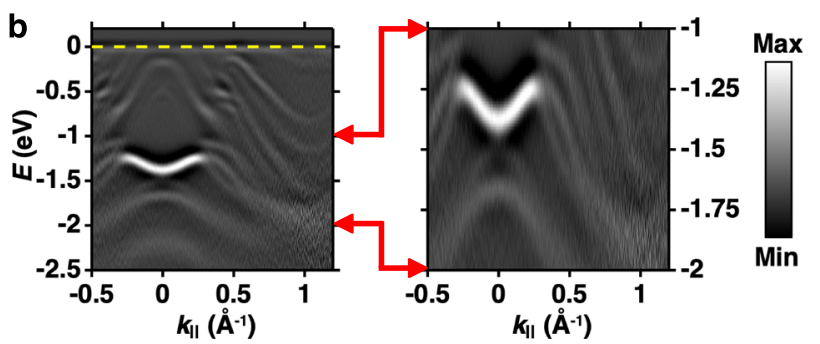

in the spectra in Figs. $3 a, b$ and $4 a, b$, we see that the number of upside-down $U$-shaped bands at the zone center in the energy range $E=-0.2$ to $-1 \mathrm{eV}$ increases by exactly one as the thickness is tuned from $3 \mathrm{TL}$ to $4 \mathrm{TL}$. However, increasing the thickness to 5 $T L$ results in an even denser set of these $U$-shaped bands, and though resolvable, it starts becoming more difficult to count these bands separately as the film is approaching the bulk limit. Corresponding statements for band counting can be duly noted for occupied bands away from the $\bar{\Gamma}$ point. Similar evolution of the band structure has been reported for the Pt-based topological semimetals ${ }^{14}$. However, unlike their Pt-based counterparts ${ }^{14}$, for all $\mathrm{NiTe}_{2}$ thin films measured here, the second-derivative ARPES maps (Figs. 2b, d, 3b, and $4 b$ ) tend to exhibit a very small amount of signal from the next layer owing to the presence of other minor thicknesses arising from the growth process, though such an effect is less apparent as the film thickness increases to $4 \mathrm{TL}$ or 5 TL. Nevertheless, when these observations are taken into account, the calculation results are in general agreement with the measured ARPES spectra, with minor discrepancies not unusual from first-principles calculations.

\section{Synchrotron Fermi surface maps for 3-5 TL $1 \mathrm{~T} \mathrm{NiTe}{ }_{2}$ films}

At last, Fig. $5 a$ and $b$ display the measured Fermi surface maps and their corresponding second derivatives for $N=3-5 \mathrm{TL} 1 \mathrm{~T} \mathrm{NiTe}_{2}$ thin films, respectively; the calculated Fermi surface maps are shown in Fig. 5c for comparison. Superficially, the three sets of experimental Fermi surface contours for $1 T \mathrm{NiTe}_{2}$ near the zone center are similar to one another in this thickness regime, but there are clear notable differences. One particular change occurs within a small, seemingly circular pocket centered about the $\bar{\Gamma}$ point, which is indicated by a white arrow in each secondderivative map in Fig. 5b. Within this circle, another small circular pocket develops prominently as the thickness increases from $3 \mathrm{TL}$ to $4 \mathrm{TL}$ (indicated by a red arrow in the $4 \mathrm{TL}$ map in Fig. 5b). Upon further increase to 5 TL in thickness, it is replaced by an even tinier pocket or dot (designated by a yellow arrow in the $5 \mathrm{TL}$ map in Fig. 5b). Aside from these changes, the Fermi surface contours themselves become denser and thus more complex with increasing film thickness, as expected for films at larger thicknesses. At some point, these contours should converge to

c
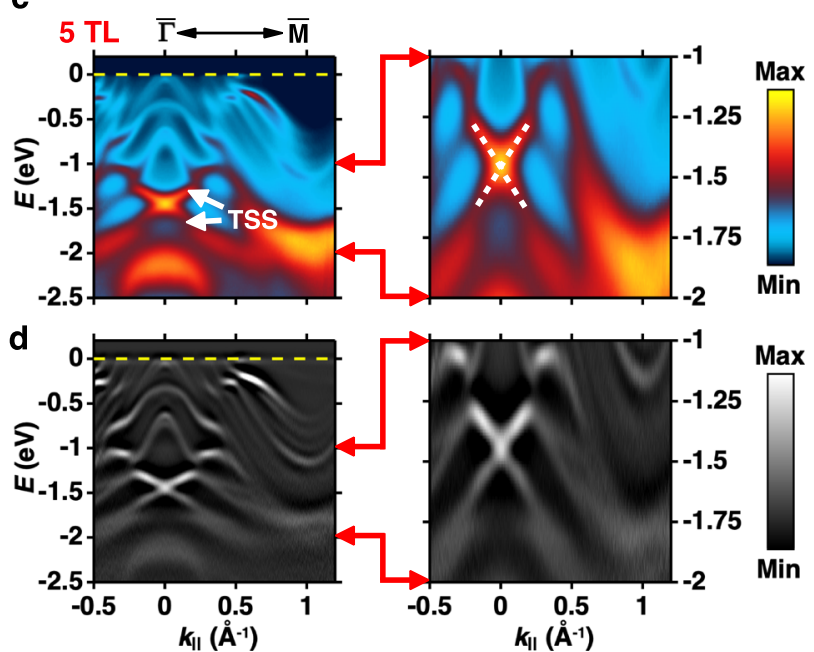

Fig. 2 Gap opening in topologically nontrivial surface states at reduced thickness. a ARPES band map of $1+2 \mathrm{TL} 1 T \mathrm{NiTe} 2$ thin film (left panel) and the same spectra zoomed-in around the conical topological surface states (right panel), which are labeled by TSS in the left panel; the data were taken with 21.218-eV photons at $30 \mathrm{~K}$. In the right panel, the white dashed curves are guides to the eye for the upper and lower branches of the gapped surface Dirac cone, and the gap opening $E_{\mathrm{g}}$ in the TSS is indicated. $\mathbf{b}$ Corresponding second-derivative ARPES results. c Overview ARPES spectra of $5 \mathrm{TL} 1 \mathrm{~T} \mathrm{NiTe}$ thin film (left panel) taken at $30 \mathrm{~K}$ with 21.218 -eV photons and the same band map zoomed-in around the topological surface states (right panel), as identified by the label TSS in the left panel. The white dashed curves are guides to the eye for the upper and lower branches for the TSS. d Corresponding second-derivative ARPES maps. 
a

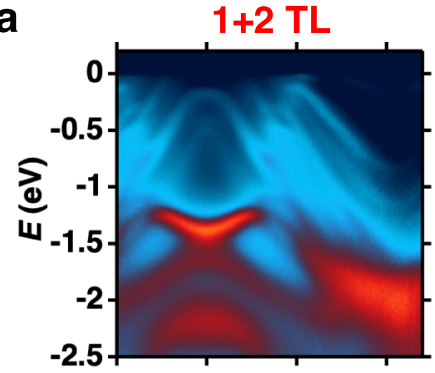

b

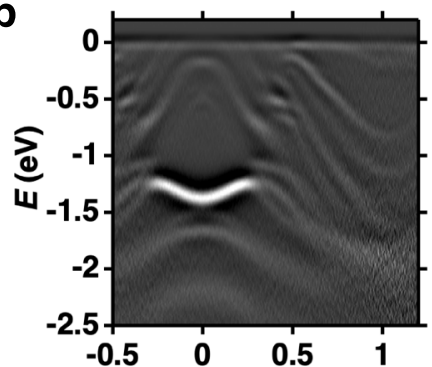

C

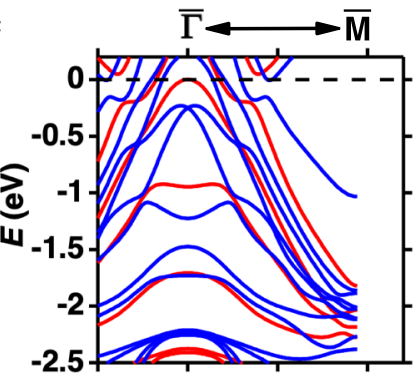

d

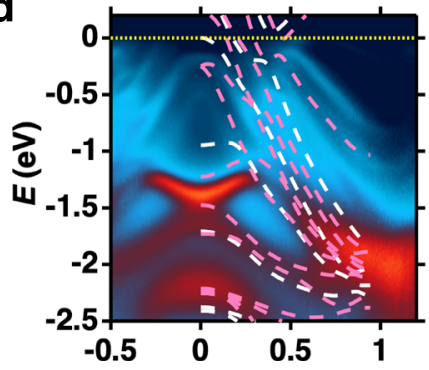

3 TL
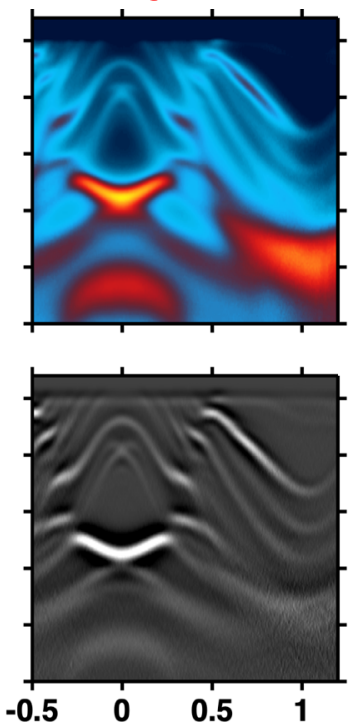

4 TL
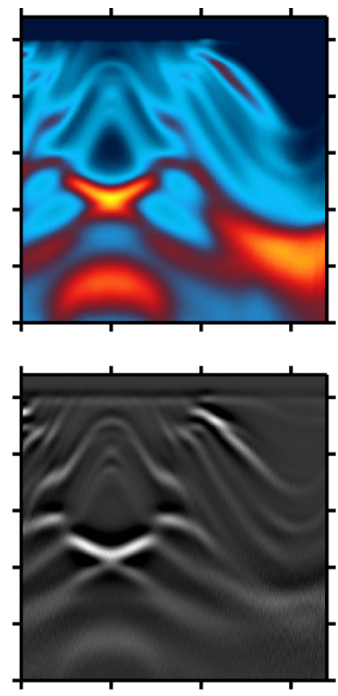

$\left(\AA^{-1}\right)$
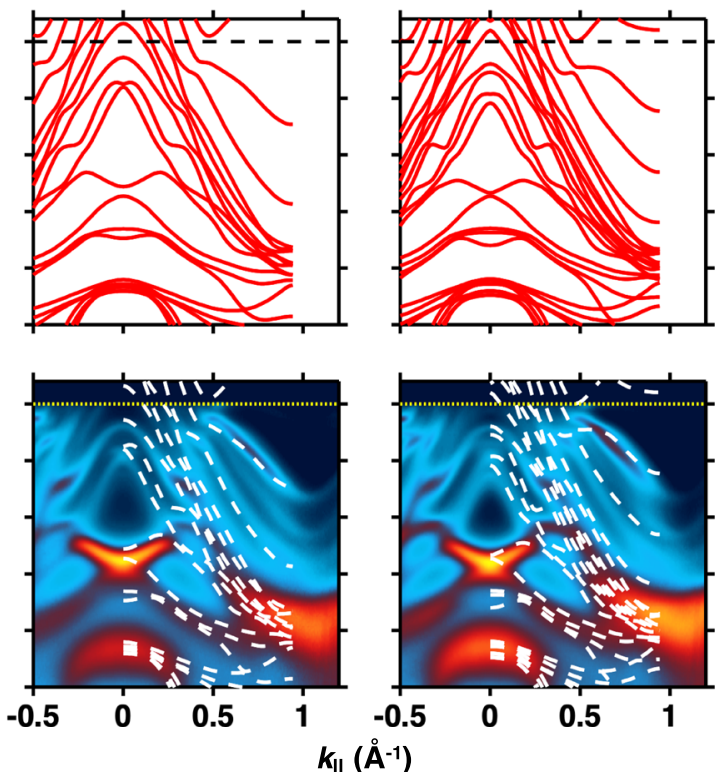

5 TL
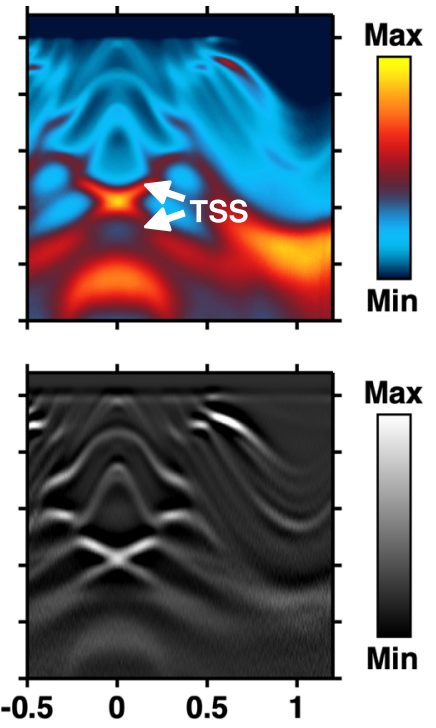

Max

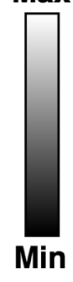

Fig. 3 Thickness-dependent ARPES spectra taken along the $\overline{\Gamma M}$ direction. a ARPES band maps of $1-5$ TL $1 T$ NiTe 2 films measured with 21.218-eV photons at $30 \mathrm{~K}$; those bands that are conical topological surface states in the bulk limit are labeled as TSS in the 5 TL map. b Corresponding second-derivative ARPES maps. c Corresponding calculated DFT band structures; in the calculation for the $1+2$ TL case, the red curves correspond to the results for a 1 TL sample, whereas the blue curves are associated with those of a 2 TL film. $\mathbf{d}$ Layer-dependent ARPES spectra from a overlaid with the same calculation results from $\mathbf{c}$; in the results for the $1+2$ TL system, the white dashed curves are associated with the 1 TL calculation and those of a 2 TL film correspond with the pink dashed curves.

the bulk Fermi surface map, modulated by ARPES matrix element effects.

These changes are further illuminating in regards to the thickness-mediated crossover of $1 T \mathrm{NiTe}_{2}$ from the ultrathin-film to a bulk-like limit reminiscent of the Type-II Dirac semimetal phase. From Figs. 3 and 4, no bands are present near the zone center at the Fermi level for a $3 \mathrm{TL}$ film, but when the thickness is increased to $4 \mathrm{TL}$, another set of hole-like bands centered about the $\bar{\Gamma}$ point develops. Evidently, this should correspondingly cause a smaller hole pocket to arise in the Fermi surface map, which is indeed seen in the 4 TL maps in Fig. 5 (indicated by the red arrow in the middle panel of Fig. 5b). A further increase in the film thickness to 5 TL causes the bands near the Fermi level at the $\bar{\Gamma}$ point to become very dense, such that the Fermi level now crosses a tiny conical band very close to its apex (see right panel of Fig. If and also Figs. 3 and 4). The experimental Fermi surface map for the $5 \mathrm{TL}$ sample should have a very tiny pocket or dot at the zone center, which is evident in the experimental result for the 5 TL film in Fig. 5 (identified by the yellow arrow in the right panel of Fig. 5b). In other words, the results for the Fermi surface maps provide additional confirmation of the crossover from the ultrathin-film to bulk-like limit as the film thickness is increased and thus corroborate with our analysis related to Figs. 3 and 4.

\section{DISCUSSION}

Our comprehensive experimental and theoretical efforts demonstrate that we have successfully fabricated ultrathin films of $1 T$ 
a

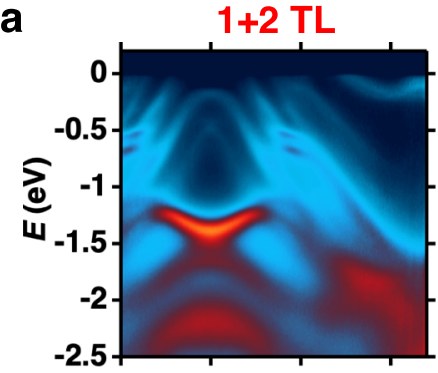

b
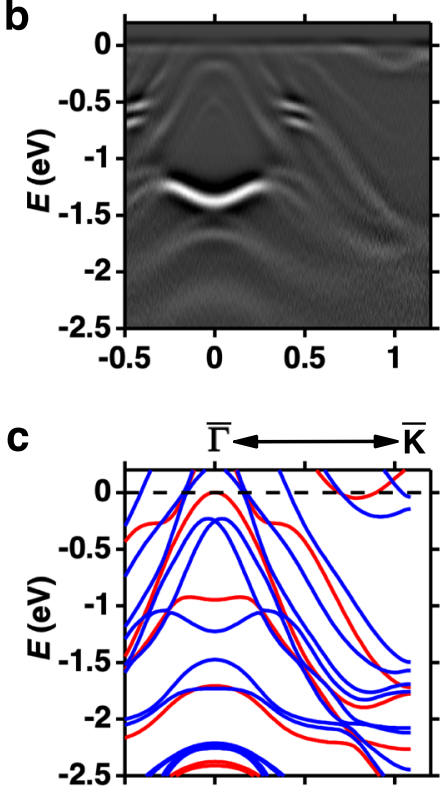

d

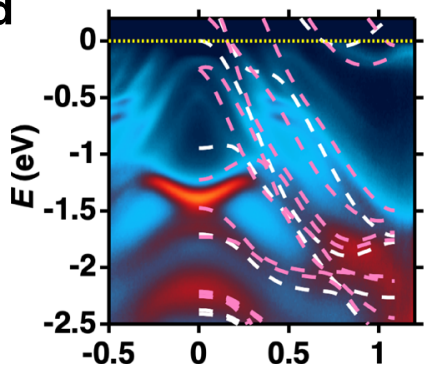

3 TL
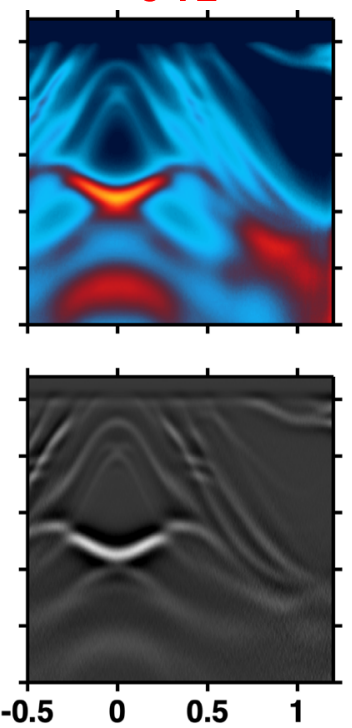

k
4 TL
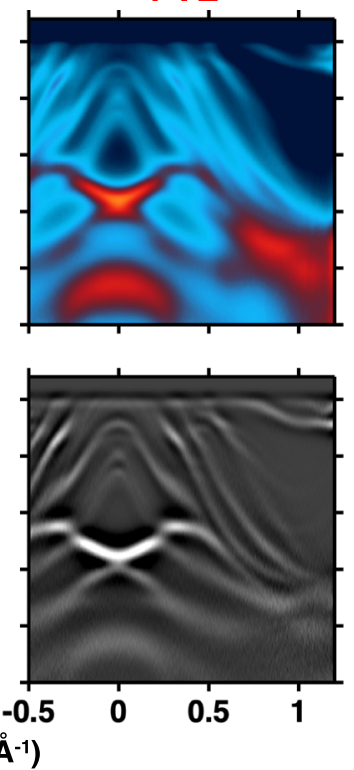
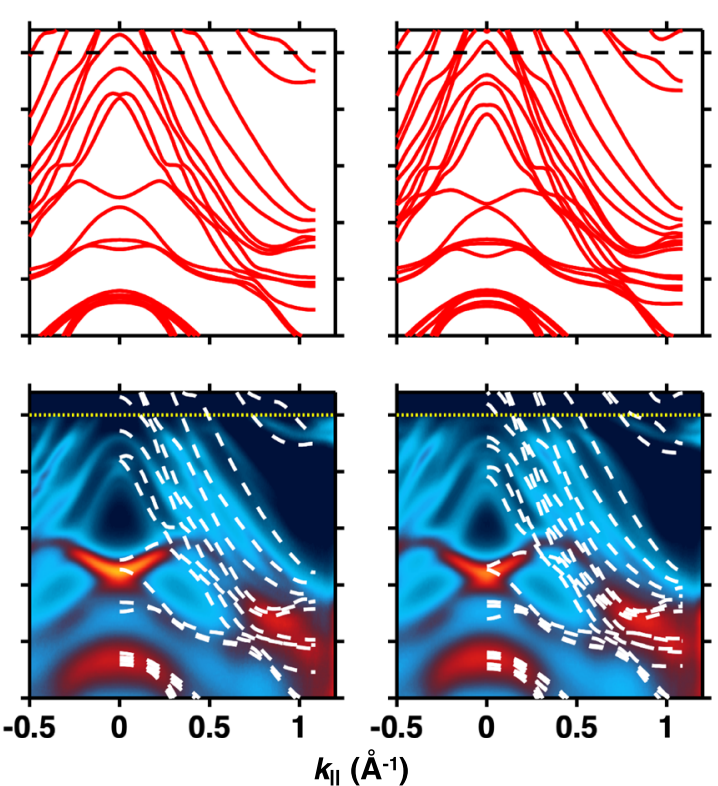

5 TL
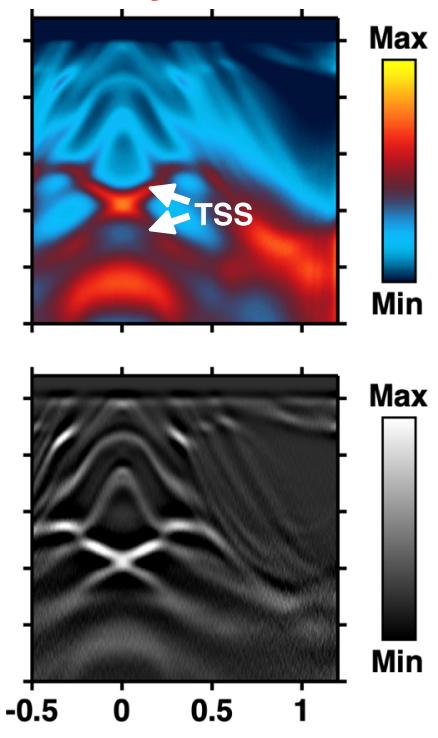
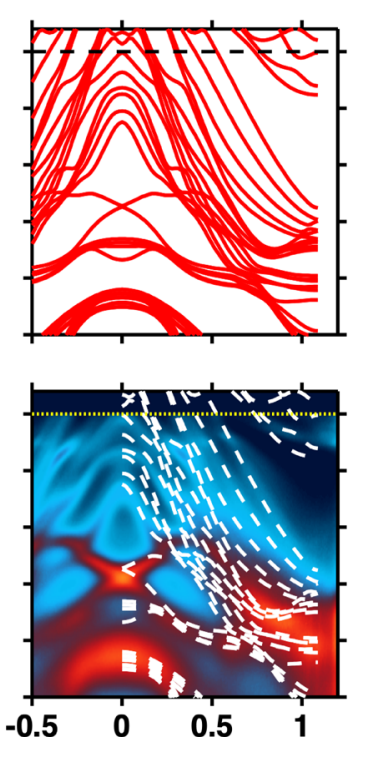

Fig. 4 Thickness-dependent ARPES maps taken along the $\overline{\Gamma K}$ direction. a ARPES band maps of 1-5 TL $1 T \mathrm{NiTe}_{2}$ films measured with $21.218-\mathrm{eV}$ photons at $30 \mathrm{~K}$; the surface bands which form a topological Dirac cone in the bulk limit are identified as TSS in the 5 TL spectra. b Corresponding second-derivative ARPES maps. c Corresponding calculated DFT results; in the calculation results for the $1+2$ TL system, the red curves are those for a $1 \mathrm{TL}$ film, and the blue curves correspond to the results for a $2 \mathrm{TL}$ film. d Thickness-dependent ARPES maps from a superimposed with the same calculation data from c; for the $1+2 \mathrm{TL}$ system, the white dashed curves are for the $1 \mathrm{TL}$ result, whereas those of a 2 TL film correspond to the pink dashed curves.

$\mathrm{NiTe}_{2}$ with thicknesses $N=1-5 \mathrm{TL}$ on BLG-SiC using MBE, a system expected to exhibit emergent, intrinsic superconductivity in the 1 TL limit and also a topological Type-II Dirac fermion phase in bulk. By employing DFT first-principles calculations, we completely dismiss any mixture of other phases, including pyrite and marcasite $\mathrm{NiTe}_{2}$, in our ARPES band maps. These results together are spectroscopic evidence for the strong thickness-mediated electronic tunability of $1 \mathrm{~T} \mathrm{NiTe}{ }_{2}$, revealing the crossover from the ultrathin-film to bulk-like limit with incipient hints of the Type-II Dirac semimetal phase with bulk Dirac fermions near the Fermi level. Our experimental ARPES maps specifically show that the hybridization gap in the topological surface Dirac cone at an energy $E \sim-1.45 \mathrm{eV}$ first closes at $5 \mathrm{TL}$ in thickness. Nevertheless, despite these successes, we discover that as-grown ultrathin $1 T$
$\mathrm{NiTe}_{2}$ films favor growing in an approximately layer-by-layer manner on BLG-SiC, suggesting enhanced interlayer coupling in this system relative to the Pt-based topological semimetals. Finally, DFT calculations and the measured ARPES spectra are in good agreement with one another. Future studies into this system may wish to instead employ local probes, such as scanning tunneling spectroscopy (STS), to characterize the electronic structure of ultrathin $1 \mathrm{~T} \mathrm{NiTe} \mathrm{N}_{2}$ films further and to confirm or

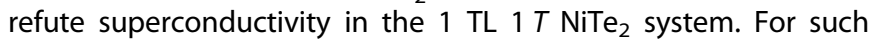
works, substrates other than BLG-SiC may also be more conducive for growth of atomically smooth $1 \mathrm{TL} 1 T \mathrm{NiTe}_{2}$ films, which would engender further assessments regarding the fabrication and suitability of $1 \mathrm{TL}$ films for technological applications. We overall expect that these findings help complete the picture for ultrathin 
a
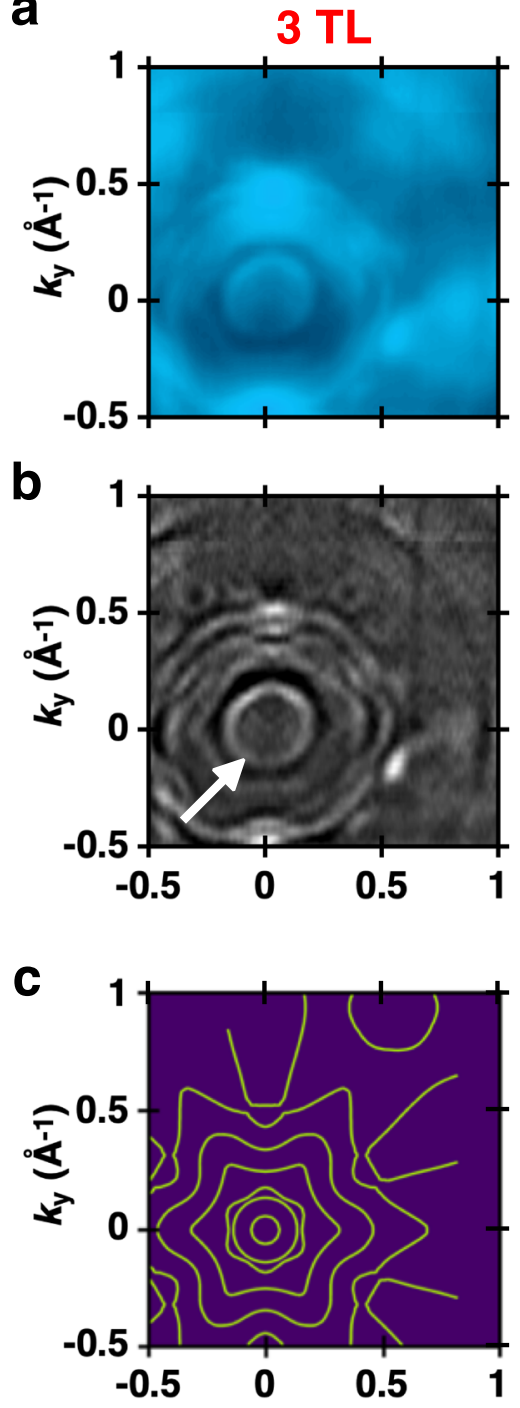
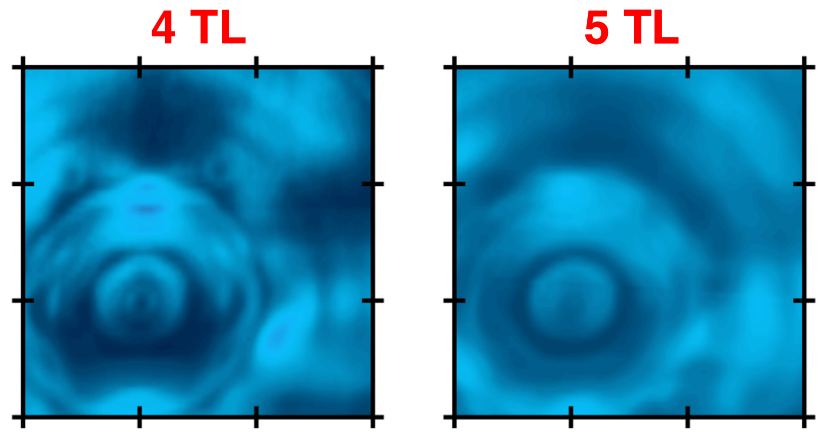

$\operatorname{Max}$
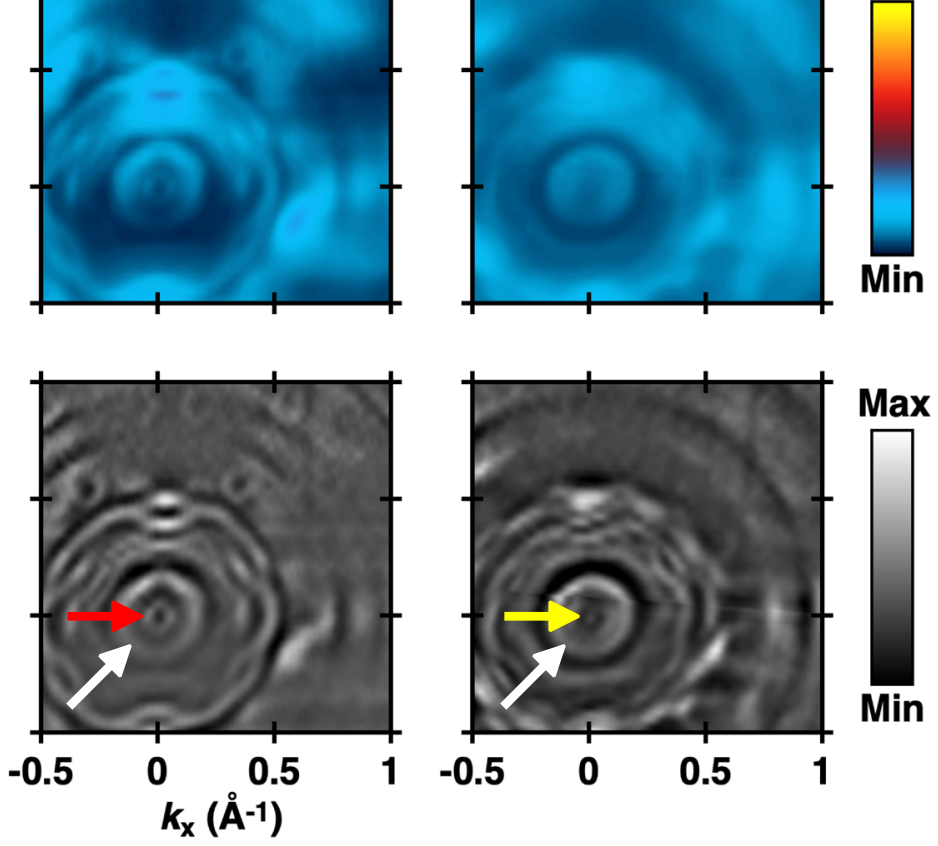

Max

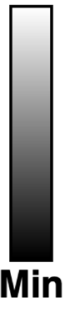

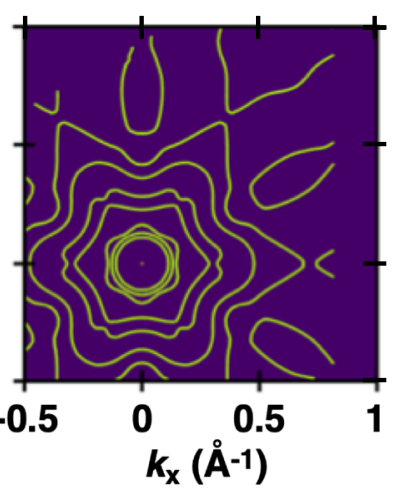

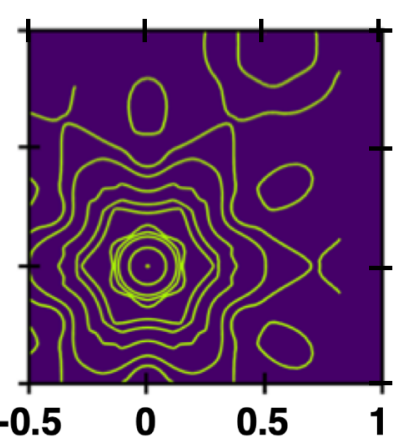

Fig. 5 Thickness-dependent Fermi surface contours for 3-5 TL 1 T NiTe $\mathbf{2}_{2}$ a Measured Fermi surface maps taken using 54-eV photons at a sample temperature of $80 \mathrm{~K}$. b Corresponding second-derivative maps. As discussed in the main text, the white arrow in each panel refers to the circular pocket centered at the zone center common to all of these film thicknesses, whereas the red and yellow arrows identify pockets distinct to the 4 TL and 5 TL systems, respectively, that arise as the thickness is varied. c Corresponding calculated Fermi surface contours; the Fermi level of the system was shifted slightly by $-0.071 \mathrm{eV}$ for better agreement with the experimental results.

films whose bulk phase is a Type-Il Dirac semimetal and also underline potential difficulties in employing MBE-grown $1 \mathrm{~T} \mathrm{\textrm {NiTe } _ { 2 }}$ films in a device setting.

\section{METHODS}

\section{Sample preparation of $1 \mathrm{~T} \mathrm{NiTe}_{2}$ films}

Prior to growth of ultrathin $\mathrm{NiTe}_{2}$ films via MBE at the University of Illinois, $6 \mathrm{H}$-SiC $(0001)$ substrates were degassed at $\sim 500^{\circ} \mathrm{C}$ for a couple hours and then flash annealed to $\sim 1300^{\circ} \mathrm{C}$ for more than 50 cycles, yielding wellordered bilayer-graphene-terminated surfaces ${ }^{41}$. High-purity $\mathrm{Ni}$ and $\mathrm{Te}$ were co-evaporated from an electron-beam evaporator ( $\mathrm{Ni}$ ) and an effusion cell (Te) onto a BLG-SiC surface maintained at $280^{\circ} \mathrm{C}$. The film growth rate was $1 \mathrm{TL}(1 \mathrm{TL} \approx 0.5 \mathrm{~nm})$ every $\sim 50 \mathrm{~min}$. Following the film growth, sample quality was confirmed in situ using RHEED and ARPES. Photoemission measurements at the University of Illinois were performed at $30 \mathrm{~K}$ using a Scienta Omicron VUV5k He discharge lamp coupled with a Scienta R4000 hemispherical analyzer. Each $\mathrm{NiTe}_{2}$ film was capped with a 10-nm-thick Te film to preserve surface integrity upon removal from the ARPES system. These samples were taken to Beamline 10.0.1.1 (HERS) at the Advanced Light Source (ALS) for additional ARPES characterizations. They were decapped in ultra-high vacuum by heating under Te flux to $\sim 300^{\circ} \mathrm{C}$ just prior to the measurements. The synchrotron results were obtained at a sample temperature of $\sim 80 \mathrm{~K}$ and an overall energy resolution better than $15 \mathrm{meV}$. In addition to the results outlined in the main discussion, constant energy contour maps $E\left(k_{\mathrm{x}}, k_{\mathrm{y}}\right)$ obtained at synchrotron for $1 T \mathrm{NiTe}_{2}$ thin films of thicknesses 3-5 TL are briefly considered in Supplementary Note 5 and Supplementary Fig. 22, whereas additional analysis and processing of data taken at the University of Illinois are detailed in Supplementary Note 6 and Supplementary Figs. 23-25.

\section{First-principles calculations}

First-principles calculations were conducted in the DFT framework ${ }^{42}$ as implemented in the Vienna ab initio simulation package ${ }^{43}$; the projected augmented wave approach was employed with an energy cutoff of $400 \mathrm{eV}$ $\left(\right.$ refs. ${ }^{44,45}$ ). To replicate the experimental lattice constants, the optB86bvdW functional ${ }^{46}$ for the van der Waals correction was used for the structural relaxation, such that the residual force on each atom was $<10^{-3} \mathrm{eV} / \AA \AA$; the self-consistent convergence criterion was set to $10^{-5} \mathrm{eV}$. Thin films were modeled as a periodic array of finite-layer systems, each 
separated from one another by a vacuum gap of $15.0 \AA \AA$. For thin films, the first Brillouin zone was sampled by employing $\Gamma$-centered Monkhorst-Pack (ref. ${ }^{47}$ ) $k$-space meshes of $24 \times 24 \times 1$, whereas for bulk structures, $k$-space meshes of $24 \times 24 \times 12,24 \times 24 \times 6$, and $24 \times 24 \times 18$ for the $1 T, 2 \mathrm{H}$, and orthorhombic (pyrite and marcasite) structures were utilized, respectively. SOC was included in the theoretical band structures presented, unless indicated otherwise. The lattice structures and select theoretical band structure results for $1 \mathrm{~T}, 2 \mathrm{H}$, pyrite, and marcasite phases of $\mathrm{NiTe}_{2}$ are considered in Supplementary Note 1 and Supplementary Figs. 1-4. Additional calculations that employ the generalized gradient approximation (GGA) with a Hubbard-like onsite Coulomb repulsion correction $(\mathrm{GGA}+\mathrm{U})\left(\right.$ refs. $\left.{ }^{48,49}\right)$ and the metaGGA SCAN functional ${ }^{50}$ for $1 T \mathrm{NiTe}_{2}$ thin films are codified in Supplementary Note 2 and Supplementary Figs. 5-10. Theoretical discussions relevant to the recently predicted superconductivity and magnetic ordering in $1 \mathrm{TL} 1 T \mathrm{NiTe}_{2}$ (refs. ${ }^{17,19}$ ) and the spin polarization of the TSS as a function of film thickness are outlined in Supplementary Notes 3 and 4, respectively, the results of which are further detailed in Supplementary Figs. 11-21 and Supplementary Tables 1-3.

\section{DATA AVAILABILITY}

All data needed to evaluate the conclusions in the paper are included in the paper and/or the Supplementary Information. Additional data related to this paper may be requested from T.C.C. (tcchiang@illinois.edu) and F.C.C. (fchuang@mail.nsysu.edu.tw).

Received: 6 September 2020; Accepted: 17 March 2021;

Published online: 12 April 2021

\section{REFERENCES}

1. Manzeli, S., Ovchinnikov, D., Pasquier, D., Yazyev, O. V. \& Kis, A. 2D transition metal dichalcogenides. Nat. Rev. Mater. 2, 17033 (2017).

2. Han, G. H., Duong, D. L., Keum, D. H., Yun, S. J. \& Lee, Y. H. Van der Waals metallic transition metal dichalcogenides. Chem. Rev. 118, 6297-6336 (2018).

3. Ataca, C., Şahin, H. \& Ciraci, S. Stable, single-layer $M_{2}$ transition-metal oxides and dichalcogenides in a honeycomb-like structure. J. Phys. Chem. C. 116, 8983-8999 (2012).

4. Mak, K. F., Lee, C., Hone, J., Shan, J. \& Heinz, T. F. Atomically thin $\operatorname{MoS}_{2}$ : a new direct-gap semiconductor. Phys. Rev. Lett. 105, 136805 (2010).

5. Zhao, W. et al. Evolution of electronic structure in atomically thin sheets of $\mathrm{WS}_{2}$ and $\mathrm{WSe}_{2}$. ACS Nano 7, 791-797 (2013).

6. Chen, P. et al. Large quantum-spin-Hall gap in single-layer $1 T^{\prime} \mathrm{WSe}_{2}$. Nat. Commun. 9, 2003 (2018)

7. Tang, S. et al. Quantum spin Hall state in monolayer $1 \mathrm{~T}^{\prime}-\mathrm{WTe} \mathrm{T}_{2}$. Nat. Phys. 13, 683-687 (2017).

8. Liu, D. et al. Electronic origin of high-temperature superconductivity in singlelayer FeSe superconductor. Nat. Commun. 3, 931 (2012).

9. Miyata, Y., Nakayama, K., Sugawara, K., Sato, T. \& Takahashi, T. High-temperature superconductivity in potassium-coated multilayer FeSe thin films. Nat. Mater. 14, 775-779 (2015).

10. Ugeda, M. M. et al. Characterization of collective ground states in single-layer $\mathrm{NbSe}_{2}$. Nat. Phys. 12, 92-97 (2016).

11. Chen, P. et al. Charge density wave transition in single-layer titanium diselenide. Nat. Commun. 6, 8943 (2015).

12. Chen, P. et al. Emergence of charge density waves and a pseudogap in singlelayer $\mathrm{TiTe}_{2}$. Nat. Commun. 8, 516 (2017).

13. Chen, P. et al. Unique gap structure and symmetry of the charge density wave in single-layer VSe 2 . Phys. Rev. Lett. 121, 196402 (2018).

14. Lin, M. K. et al. Dimensionality-mediated semimetal-semiconductor transition in ultrathin PtTe 2 films. Phys. Rev. Lett. 124, 036402 (2020).

15. Zhao, B. et al. Synthetic control of two-dimensional $\mathrm{NiTe}_{2}$ single crystals with highly uniform thickness distributions. J. Am. Chem. Soc. 140, 14217-14223 (2018).

16. Shi, J. et al. Two-dimensional metallic $\mathrm{NiTe}_{2}$ with ultrahigh environmental stability, conductivity, and electrocatalytic activity. ACS Nano 14, 9011-9020 (2020).

17. Zheng, F. et al. Emergent superconductivity in two-dimensional $\mathrm{NiTe}_{2}$ crystals. Phys. Rev. B 101, 100505(R) (2020).

18. Feng, Z. et al. Evidences for pressure-induced two-phase superconductivity and mixed structures of $\mathrm{NiTe}_{2}$ and NiTe in type-II Dirac semimetal $\mathrm{NiTe}_{2-\mathrm{x}}(x=0.38 \pm$ 0.09) single crystals. Mater. Today Phys. 17, 100339 (2021).

19. Aras, M., Kıllıç, Ç. \& Ciraci, S. Magnetic ground state in $\mathrm{FeTe}_{2}, \mathrm{VS}_{2}$, and $\mathrm{NiTe}_{2}$ monolayers: antiparallel magnetic moments at chalcogen atoms. Phys. Rev. B 101, 054429 (2020).
20. Dulal, R. P. et al. Nanostructures of type-II topological Dirac semimetal $\mathrm{NiTe}_{2}$. J. Vac. Sci. Technol. B 37, 042903 (2019).

21. Xu, C. et al. Topological Type-II Dirac fermions approaching the Fermi level in a transition metal dichalcogenide $\mathrm{NiTe}_{2}$. Chem. Mater. 30, 4823-4830 (2018).

22. Liu, Q. et al. Nontopological origin of the planar Hall effect in the type-II Dirac semimetal $\mathrm{NiTe}_{2}$. Phys. Rev. B 99, 155119 (2019).

23. Ghosh, B. et al. Observation of bulk states and spin-polarized topological surface states in transition metal dichalcogenide Dirac semimetal candidate $\mathrm{NiTe}_{2}$. Phys. Rev. B 100, 195134 (2019).

24. Mukherjee, S. et al. Fermi-crossing Type-II Dirac fermions and topological surface states in $\mathrm{NiTe}_{2}$. Sci. Rep. 10, 12957 (2020).

25. Bahramy, M. S. et al. Ubiquitous formation of bulk Dirac cones and topological surface states from a single orbital manifold in transition-metal dichalcogenides. Nat. Mater. 17, 21-28 (2018).

26. Clark, O. J. et al. Fermiology and superconductivity of topological surface states in PdTe $_{2}$. Phys. Rev. Lett. 120, 156401 (2018).

27. Noh, H. J. et al. Experimental realization of Type-II Dirac fermions in a $\mathrm{PdTe}_{2}$ superconductor. Phys. Rev. Lett. 119, 016401 (2017).

28. Yan, M. et al. Lorentz-violating type-II Dirac fermions in transition metal dichalcogenide $\mathrm{PtTe}_{2}$. Nat. Commun. 8, 257 (2017).

29. Zhang, K. et al. Experimental evidence for type-II Dirac semimetal in PtSe 2 . Phys. Rev. B 96, 125102 (2017)

30. Politano, A. et al. 3D Dirac plasmons in the Type-II Dirac semimetal PtTe 2 . Phys. Rev. Lett. 121, 086804 (2018).

31. Zheng, W. et al. Detailed study of the Fermi surfaces of the type-II Dirac semimetallic candidates $X \mathrm{Te}_{2}(X=\mathrm{Pd}, \mathrm{Pt})$. Phys. Rev. B 97, 235154 (2018).

32. Udagawa, M. \& Bergholtz, E. J. Field-selective anomaly and chiral mode reversal in Type-II Weyl materials. Phys. Rev. Lett. 117, 086401 (2016).

33. Alidoust, M., Halterman, K. \& Zyuzin, A. A. Superconductivity in type-II Weyl semimetals. Phys. Rev. B 95, 155124 (2017).

34. Villaos, R. A. B. et al. Thickness dependent electronic properties of Pt dichalcogenides. NPJ 2D Mater. Appl. 3, 2 (2019).

35. Chia, X., Sofer, Z., Luxa, J. \& Pumera, M. Unconventionally layered CoTe 2 and $\mathrm{NiTe}_{2}$ as electrocatalysts for hydrogen evolution. Chem. Eur. J. 23, 11719-11726 (2017).

36. Bensch, W. et al. Anionic polymeric bonds in nickel ditelluride: Crystal structure, and experimental and theoretical band structure. J. Solid State Chem. 121, 87-94 (1996).

37. Jobic, S., Brec, R. \& Rouxel, J. Anionic polymeric bonds in transition metal ditellurides. J. Solid State Chem. 96, 169-180 (1992).

38. Liu, Y., Bian, G., Miller, T., Bissen, M. \& Chiang, T. C. Topological limit of ultrathin quasi-free-standing $\mathrm{Bi}_{2} \mathrm{Te}_{3}$ films grown on Si(111). Phys. Rev. B 85, 195442 (2012).

39. Zhang, Y. et al. Crossover of the three-dimensional topological insulator $\mathrm{Bi}_{2} \mathrm{Se}_{3}$ to the two-dimensional limit. Nat. Phys. 6, 584-588 (2010).

40. Chiang, T. C. Photoemission studies of quantum well states in thin films. Surf. Sci. Rep. 39, 181-235 (2000).

41. Wang, Q. et al. Large-scale uniform bilayer graphene prepared by vacuum graphitization of $6 \mathrm{H}-\mathrm{SiC}(0001)$ substrates. J. Phys. Condens. Matter 25, 095002 (2013).

42. Hohenberg, P. \& Kohn, W. Inhomogeneous electron gas. Phys. Rev. 136, B864-B871 (1964).

43. Kresse, G. \& Hafner, J. Ab initio molecular dynamics for liquid metals. Phys. Rev. $B$ 47, 558(R)-561(R) (1993).

44. Blöchl, P. E. Projector augmented-wave method. Phys. Rev. B 50, 17953-17979 (1994).

45. Kresse, G. \& Joubert, D. From ultrasoft pseudopotentials to the projector augmented-wave method. Phys. Rev. B 59, 1758-1775 (1999).

46. Klimeš, J., Bowler, D. R. \& Michaelides, A. Chemical accuracy for the van der Waals density functional. J. Phys. Condens. Matter 22, 022201 (2010).

47. Monkhorst, H. J. \& Pack, J. D. Special points for Brillouin-zone integrations. Phys. Rev. B 13, 5188-5192 (1976).

48. Perdew, J. P., Burke, K. \& Ernzerhof, M. Generalized gradient approximation made simple. Phys. Rev. Lett. 77, 3865-3868 (1996).

49. Dudarev, S. L., Botton, G. A., Savrasov, S. Y., Humphreys, C. J. \& Sutton, A. P. Electron-energy-loss spectra and the structural stability of nickel oxide: An LSDA+U study. Phys. Rev. B 57, 1505-1509 (1998).

50. Sun, J., Ruzsinszky, A. \& Perdew, J. P. Strongly constrained and appropriately normed semilocal density functional. Phys. Rev. Lett. 115, 036402 (2015).

\section{ACKNOWLEDGEMENTS}

This work was supported by the U.S. Department of Energy (DOE), Office of Science (OS), Office of Basic Energy Sciences, Division of Materials Science and Engineering, under grant no. DE-FG02-07ER46383 (T.C.C.). F.C.C. acknowledges support from the National Center for Theoretical Sciences and the Ministry of Science and Technology of Taiwan under grants no. MOST-107-2628-M-110-001-MY3. He is also grateful to the National Center for High-Performance Computing for computer time and facilities. This research used resources of the ALS, which is a DOE Office of Science User Facility under Contract No. DE-AC02-05CH11231. 


\section{AUTHOR CONTRIBUTIONS}

J.A.H., M.K.L., and T.C.C., with the aid of Y.L., performed the MBE growths. L.Y.F. and F.C.C. with R.A.B.V. performed the first-principles calculations. J.A.H., M.K.L., and T.C.C., with the help of R.Y.L., P.C., and S.K.M., conducted the synchrotron measurements and/or ARPES characterizations at the University of Illinois. J.A.H., M.K.L., and T.C.C. analyzed the data. J.A.H. and T.C.C. wrote the paper. T.C.C., F.C.C., J.A.H., L.Y.F., M.K.L., and R.A.B.V. interpreted the data. J.A.H., L.Y.F., and M.K.L. contributed equally and are co-first authors; T.C.C. and F.C.C. are the corresponding authors. T.C.C. led the experimental group; F.C.C. led the theory group. T.C.C. organized the project.

\section{COMPETING INTERESTS}

The authors declare no competing interests.

\section{ADDITIONAL INFORMATION}

Supplementary information The online version contains supplementary material available at https://doi.org/10.1038/s41699-021-00218-z.

Correspondence and requests for materials should be addressed to F.-C.C. or T.-C.C.
Reprints and permission information is available at http://www.nature.com/ reprints

Publisher's note Springer Nature remains neutral with regard to jurisdictional claims in published maps and institutional affiliations.

Open Access This article is licensed under a Creative Commons Attribution 4.0 International License, which permits use, sharing, adaptation, distribution and reproduction in any medium or format, as long as you give appropriate credit to the original author(s) and the source, provide a link to the Creative Commons license, and indicate if changes were made. The images or other third party material in this article are included in the article's Creative Commons license, unless indicated otherwise in a credit line to the material. If material is not included in the article's Creative Commons license and your intended use is not permitted by statutory regulation or exceeds the permitted use, you will need to obtain permission directly from the copyright holder. To view a copy of this license, visit http://creativecommons org/licenses/by/4.0/.

(c) The Author(s) 2021 